\title{
Evaluation of the Tensile Strength of Electron Beam Irradiated Powdered Ultra High Molecular Weight-Polyethylene (UHMWPE) Prior to Sintering
}

\author{
Masae Kanda ${ }^{1}$, Tiana Deplancke ${ }^{2}$, Olivier Lame ${ }^{2}$, Yoshitake Nishi ${ }^{1}$ and Jean-Yves Cavaille ${ }^{2}$ \\ ${ }^{1}$ Department of Metallurgical Engineering, Graduate School of Engineering, Tokai University, Hiratsuka 259-1292, Japan \\ ${ }^{2}$ MATEIS, UMR CNRS-INSA Lyon, Université de Lyon, 69621 Villeurbanne, France
}

\begin{abstract}
We have studied the variation in tensile strength $\left(\sigma_{\mathrm{b}}\right)$, fracture strain $\left(\varepsilon_{\mathrm{f}}\right)$ and Young modulus $(\mathrm{d} \sigma / \mathrm{d} \varepsilon)$ of low voltage electron beam irradiation (HLEBI) on powdered ultra-high molecular weight-polyethylene from 0.043 to $0.43 \mathrm{MGy}$ prior to sintering. The low dose of $0.043 \mathrm{MGy}-\mathrm{HLEBI}$ enhanced the $\sigma_{\mathrm{b}}, \varepsilon_{\mathrm{f}}$ and $\mathrm{d} \sigma / \mathrm{d} \varepsilon$, as well as increased the crystallinity ratio from 37 to $46 \%$ evaluated by differential scanning calorimetry (DSC). An additional dose apparently decreased the $\sigma_{\mathrm{b}}$ and $\varepsilon_{\mathrm{f}}$, as well as the melting point from approximately 412 to $406 \mathrm{~K}$ and the crystallinity ratio from 46 to $43 \%$ for 0.043 to $0.43 \mathrm{MGy}$, respectively. Irradiation induces two competitive mechanisms, namely (i) the scission of chains and (ii) the crosslinking of chains. The discussion is focused on these mechanisms. [doi:10.2320/matertrans.M2015108]
\end{abstract}

(Received March 13, 2015; Accepted June 18, 2015; Published July 27, 2015)

Keywords: ultra high molecular weight $(U H M W)$-polyethylene, electron beam irradiation, tensile test

\section{Introduction}

Polyethylene (PE), is one of the simplest of all commercial polymers made from the ethylene monomer $\left(-\mathrm{CH}_{2}-\right)$, is the most popular polymer, which is utilized for grocery bags, shampoo bottles, children's toys, and even bullet proof vests for ultra high molecular weight PE. A PE molecule is nothing more than a long chain of carbon atoms, with two hydrogen atoms attached to each carbon atom, only with the chain of carbon atoms being many thousands of atoms long. Increasing mean molecular weight and chain perfection generally increases chemical resistance and mechanical Young modulus.

However, ultra-high molecular weight polymers display a very high viscosity that prevents its processing by conventional injection or extrusion. Indeed, polymer viscosity and reptation time are related to the molecular weight at the power 3 or close to. ${ }^{1,2)}$ In contrast, high molecular weight generally results in high mechanical performances, particularly regarding the resistance to creep, impact and wear. Due to its performance and versatility the most commonly used high molecular mass polymer is polyethylene. The latter is processed only by compression-molding, ram-extrusion or sintering of nascent powders. ${ }^{3-5)}$

To improve the fracture toughness to insure materials safety and performances, sintering has to be optimized. One possible route is to enhance the particle/particle strength by applying a $100 \mathrm{keV}$-class low voltage electron beam irradiation (HLEBI) to surfaces of the ultra-high molecular weight-polyethylene (UHMWPE). HLEBI is generally one of the possible method to improve wear resistance, burnishing, change wetting, ${ }^{6}$ and sterilization. ${ }^{7)}$ The extremely high stability and homogeneity of industrial HLEBI equipments have been practically utilized for wallpaper sheet up to several square meters in size. Furthermore, HLEBI has induced the mist resistance, ${ }^{8-10)}$ Young modulus, strength, its strain and fracture strain for practical use of epoxy and polycarbonate. ${ }^{11,12)}$

Since the dangling bonds generated by the low-voltage electron beam should act as bonding sites in UHMWPE,
HLEBI is expected to raise its strength. In this paper, the strategy was first to use HLEBI on the nascent powders of UHMWPE $(\mathrm{Mw}=10.5 \mathrm{Mg} / \mathrm{mol})$ then to sinter the polymer powders above the melting point $(473 \mathrm{~K})$. Finally, the purpose of the present work is to investigate the effects of HLEBI on the tensile mechanical properties.

\section{Experimental Procedure}

\subsection{Condition of EB-irradiation}

The material used is the nascent powder of UHMWPE, GUR 4170, produced by TICONA, with the molecular weight $10.5 \mathrm{Mg} / \mathrm{mol}$. The powders were treated by the prototype equipment known as the electron-curtain processor (Type CB175/15/180L, Energy Science Inc., Woburn, MA), which contain a vacuum chamber with a tungsten filament and a thin titanium window, and sample treatment compartment with conveyer under 1 atm $\mathrm{N}_{2}$ gas. ${ }^{8-12)}$ The tungsten filament in vacuum was used to generate the electron beam at a low energy (acceleration potential, $V$ of $170 \mathrm{kV}$ and irradiating current density $J$ of $0.089 \mathrm{Am}^{-2}$ ). Although the electron beam generation was under vacuum, the irradiated sample was kept under protective $\mathrm{N}_{2}$ gas. The gas flow rate was $1.51 \cdot \mathrm{s}^{-1}$ at $0.1 \mathrm{MPa}$ of $\mathrm{N}_{2}$ gas pressure with an $\mathrm{O}_{2}$ residual concentration below $400 \mathrm{ppm}$. The sheet electron beam irradiation was applied intermittently. Each sweep of irradiation dose (0.043 MGy $\left.\left(\mathrm{kJ} \mathrm{g}^{-1}\right)\right)$ was applied for only a short time $(0.23 \mathrm{~s})$ to avoid excessive heating of the sample; the temperature of the sample surface remained below $323 \mathrm{~K}$ just after irradiation. Repeated irradiations to both side surfaces of the samples with the interval of $30 \mathrm{~s}$ were used to increase the total dose of irradiation. The UHMWPE powder sample was treated by HLEBI on both outside surfaces only. Given the densities $(\rho)$ was $0.97 \mathrm{Mg} \cdot \mathrm{m}^{-3}\left(\mathrm{~g} \cdot \mathrm{cm}^{-3}\right)$ for PE, the penetration depth $\left(D_{\text {th }}\right)$ values of $0.27 \mathrm{~mm}$ for PE were estimated by assumptions of Christenhusz and Reimer, respectively. ${ }^{13)}$ In addition, the $D_{\text {th }}$ value of PE $(0.4 \mathrm{~mm})$ was also calculated by the assumptions of Libby. ${ }^{14)}$ Namely, the effective depth of homogeneous irradiation was $0.3 \pm$ $0.1 \mathrm{~mm}$. 


\subsection{Sample preparation and tensile test}

After EB-irradiation treatment, the UHMWPE powder was rapidly sintered under pressure at $473 \mathrm{~K}$ during $1 \mathrm{~h}$ in an Instron compressive machine equipped with a Servantin oven. The sintering protocol is detailed in the reference. ${ }^{5)}$ Sintered disc with $2 \mathrm{~mm}$ in thickness and $25 \mathrm{~mm}$ of diameter was obtained. Each sintered sample provides 2 tensile specimens with $10 \mathrm{~mm}$ in gauge length, $2 \mathrm{~mm}$ in thickness and $3 \mathrm{~mm}$ of width, hence the cross-sectional area was $6 \mathrm{~mm}^{2}$.

The UHMWPE is known to have a very broad rubbery plateau. This exceptional property permits to perform the tensile test above as well as below the melting temperature. ${ }^{5)}$ All tensile tests have been performed using a tensile machine MTS 1/ME equipped with MTS CE412 oven.

For the tensile tests at room temperature the cross-head speed was $2 \mathrm{~mm} / \mathrm{min}$, thus the initial strain rate was $3.5 \times 10^{-3} \mathrm{~s}^{-1}$. For the tensile tests at $423 \mathrm{~K}$ (above melting temperature), the cross-head speed was $6 \mathrm{~mm} / \mathrm{min}$, thus the initial strain rate was $10^{-2} \mathrm{~s}^{-1}$. The nominal strain was computed as $\varepsilon=L / L_{0}$ where $L_{0}$ is the initial sample gauge length and $L$ its length under strain. The nominal stress was defined as $\sigma=F / S_{0}$ where $F$ is the tensile force and $S_{0}$ the initial sample cross-section.

\subsection{Characterization}

The crystallinity and the melting temperature was measured by Differential scanning calorimetry (DSC) analysis using a PerkinElmer Pyris apparatus at heating rate of $10 \mathrm{~K} / \mathrm{min}$. Temperature and heat flow scales were calibrated using highpurity indium. X-ray diffraction (XRD: D8 ADVANCE, BRUKER $)^{15)}$ was used to evaluate the crystallinity ratio $\chi$ obtained for UHMWPE, as received or treated by HLEBI. The experimental error of energy was within less than $2 \%$.

\section{Results}

\subsection{Stress-Strain curves of tensile test of UHMWPE with and without HLEBI}

Effects of HLEBI from 0.043 to $0.43 \mathrm{MGy}$ (performed prior to sintering) on the tensile strength $\left(\sigma_{\mathrm{b}}\right)$, fracture strain $\left(\varepsilon_{\mathrm{f}}\right)$, yield strength $\left(\sigma_{\mathrm{y}}\right)$ and Young modulus $(\mathrm{d} \sigma / \mathrm{d} \varepsilon)$ of sintered UHMWPE have been investigated. Figure 1 shows stress-strain curves of tensile test of UHMWPE without irradiation and with HLEBI from 0.043 to $0.43 \mathrm{MGy}$ (a) at room temperature and (b) above the melting point at $423 \mathrm{~K}$. Each tensile test is repeated two times independently.

As shown in Fig. 1(a), the high dose of 0.43 MGy-HLEBI decreases the ductility, as expected because of radiation damages. It is one forth that of untreated UHMWPE. On the contrary, the low dose with 0.043 MGy-HLEBI slightly increases the $\sigma_{\mathrm{b}}$ and $\varepsilon_{\mathrm{f}}$, as well as enhances the Young modulus indicated by $\mathrm{d} \sigma / \mathrm{d} \varepsilon$, which is 2 times higher than that of untreated UHMWPE. Thus the strengthening of UHMWPE induced by HLEBI is clearly shown. 0.22 MGyHLEBI induces a rather large damage of the polymer, decreasing by $50 \%$ the strain at break and by $20 \%$ the yield stress.

Moreover, strong effects of HLEBI on tensile mechanical properties at $423 \mathrm{~K}$ are observed. The elongation at break is drastically decreased which clearly correspond to the scission
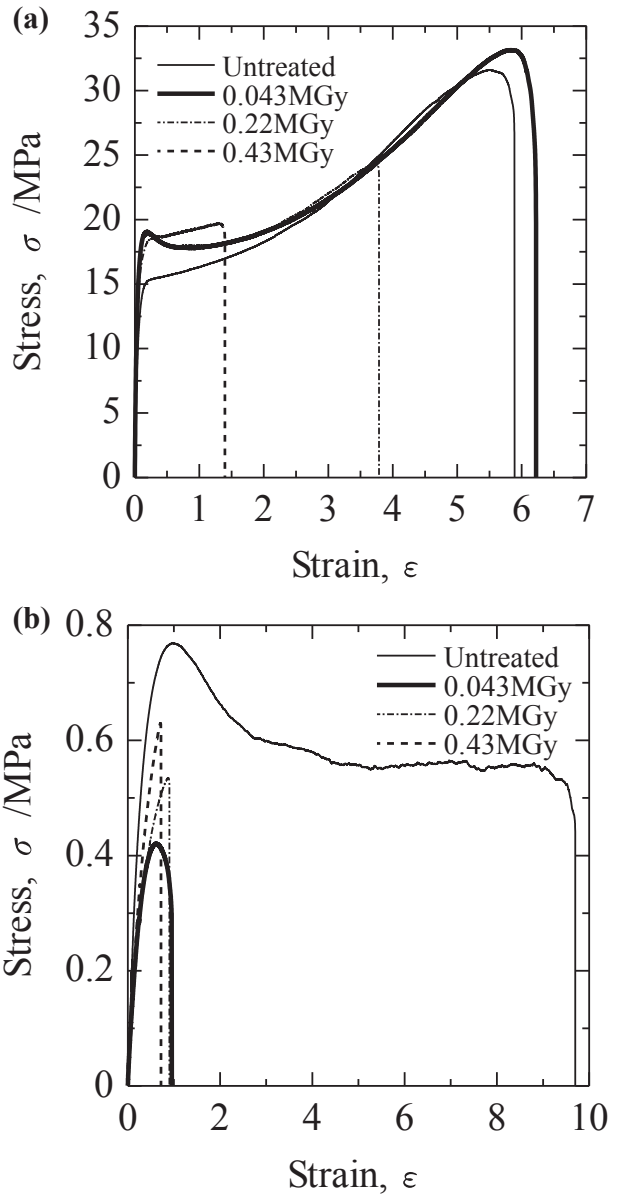

Fig. 1 Stress-strain curves of tensile test of UHMWPE sintered with and without HLEBI from 0.043 to $0.43 \mathrm{MGy}$ HLEBI dose at (a) room temperature and (b) $423 \mathrm{~K}$.

of macromolecules, because at this temperature all crystallites have melted and the material acts as a rubber.

\subsection{Effect of HLEBI on Young modulus, strength and fracture strain}

The Young modulus $(\mathrm{d} \sigma / \mathrm{d} \varepsilon)$ was computed from a linear regression on the experimental data in the strain range $0.01<\varepsilon<0.03$ to avoid experimental errors from both strain onset determination and viscoelastic deformation. The fracture strain $\left(\varepsilon_{\mathrm{f}}\right)$ is given as a percentage of the original gauge length.

Figure 2 shows changes in the Young modulus and fracture strain of tensile test of UHMWPE sintered against HLEBI dose from 0.043 to $0.43 \mathrm{MGy}$. The data points refer to average values of two independent measurements.

\section{Discussion}

\subsection{XRD discussion}

Figure 3 exhibits the XRD peaks of UHMWPE with and without HLEBI. The top and second highest peaks of all UHMWPE samples are observed at about 21.5 and 23.9 degree (classical orthorhombic phase $\mathrm{PE}^{16)}$ ).

\subsection{DSC analysis of HLEBI effect}

Figure 4 shows DSC analysis of UHMWPE sintered with and without HLEBI from 0.043 to 0.43 MGy HLEBI dose. 


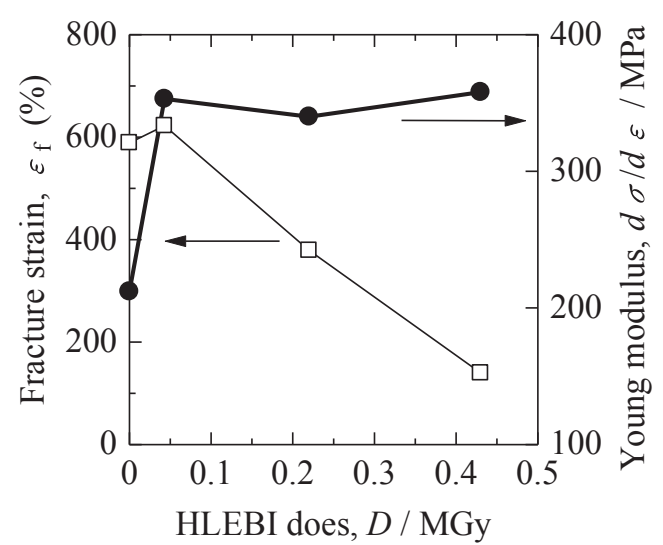

Fig. 2 Changes of the Young modulus $(\mathrm{d} \sigma / \mathrm{d} \varepsilon)$ and fracture strain $\left(\varepsilon_{\mathrm{f}}\right)$ of tensile test of sintered UHMWPE against HLEBI dose from 0.043 to 0.43 MGy.

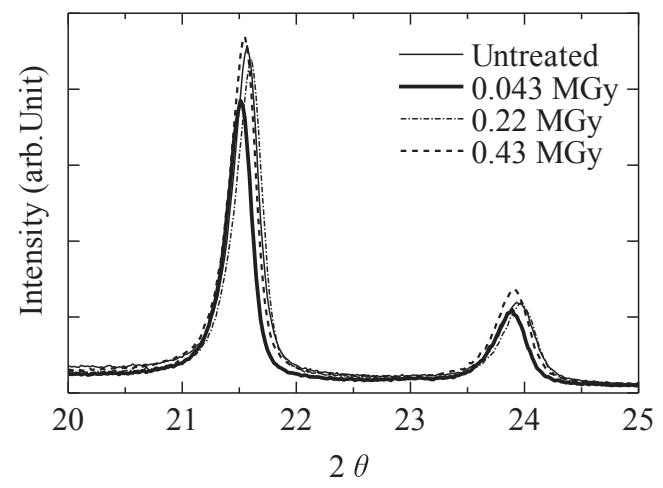

Fig. 3 Examples of XRD peaks of UHMWPE with and without HLEBI.

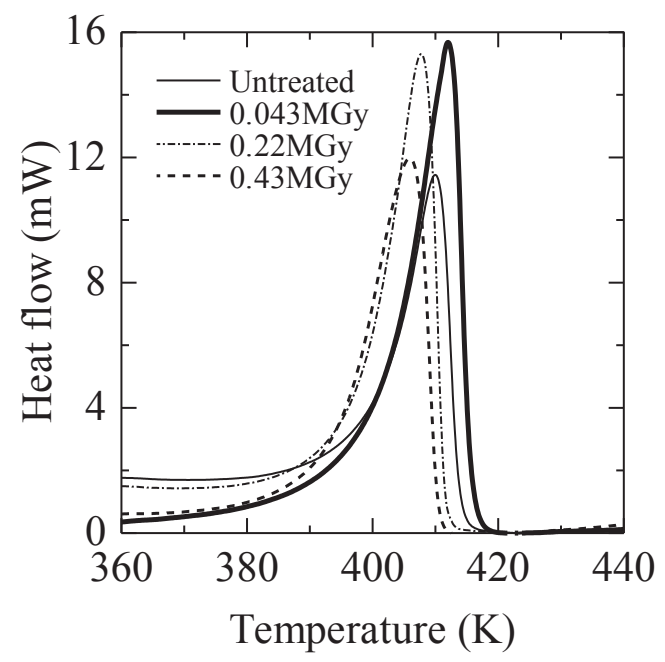

Fig. 4 DSC analysis of UHMWPE sintered with and without HLEBI from 0.043 to 0.43 MGy HLEBI dose.

Table 1 shows melting point and crystalline ratio measured by DSC.

The melting point and its enthalpy values $(412 \mathrm{~K}, 132 \mathrm{~J} / \mathrm{g})$ of $0.043 \mathrm{MGy}$ sample is 1.24 times higher than that $(410 \mathrm{~K}$, $107 \mathrm{~J} / \mathrm{g}$ ) of untreated sample. Although the crystalline ratio is $37 \%$ for untreated sample, HLEBI with low dose of $0.043 \mathrm{MGy}$ increases the crystalline ratio of $46 \%$. In the case of HLEBI with additional high dose of $0.43 \mathrm{MGy}$, the crystallinity ratio slightly decreases to $43 \%$.
Table 1 Melting point (M.P./K) and crystallinity ratio $(\chi / \%)$ at each dose of HLEBI (MGy).

\begin{tabular}{ccccc}
\hline Dose (MGy) & Untreated & 0.043 & 0.22 & 0.43 \\
\hline M.P. (K) & 410 & 412 & 408 & 406 \\
$\chi(\%)$ & 37 & 46 & 46 & 43 \\
\hline
\end{tabular}

\subsection{Atomic scale discussion of HLEBI strengthening of} Young modulus, strength and fracture strain

When tensile tests are performed at room temperature, the cohesion of the materials originates essentially from the macromolecule entanglements and crystallites in the region around native particle interface. In addition, possible crosslinking could occur due to HLEBI treatment. On the other hand, only entanglements and possible cross-linking play a role, when the tensile tests are performed above the melting point.

The Young modulus enhancement can be essentially explained by the crystallinity evolutions. The increase of crystallinity ratio is generally attributed to an increase of the chain mobility allowed by the decrease of the chain length induced by irradiation. But the very little final decrease of crystallinity ratio for high dose can be explained by the appearance of defects, such as cross-linking between to chain segments preventing large crystal growth. Thus, two mechanisms are in competition, (i) chain cuts and (ii) crosslinking of PE macromolecules with two different kinetics. ${ }^{17,18)}$ The melting temperature is generally related to the crystalline size. ${ }^{19)}$ The melting temperature increases in the case of HLEBI dose 0.043 compared to the untreated material.

According to Table 1, a low dose leads to an increase of $\chi$ from 37 to $46 \%$ which in turn is enough to explain the experimental mechanical data. Indeed, the decrease of the chain length in this region increases the macromolecule mobility allowing the polymer to further crystallize such as classical linear PE (i.e. with shorter chains). This hypothesis is consistent with the intensity increase of the XRD peak, the area under which is directly proportional to the crystalline content. Moreover, it is known that mechanical properties of semi-crystalline polymers such as $\mathrm{d} \sigma / \mathrm{d} \varepsilon$ and $\sigma_{\mathrm{y}}$ are directly correlated to the crystallinity.

On the other hand, it is well known that irradiation leads to changes of the distance between first neighbors, enhancing the first coordinated atomic distance, resulting in turn in atomic scale volume expansion by dangling bonds formation. Such a situation is well known for various polymers in their glassy state, such as epoxy, ${ }^{20)}$ polycarbonate, ${ }^{21)}$ acryl resins ${ }^{22)}$ as well as Silicate glasses. ${ }^{23-26)}$ Therefore, the high $\mathrm{d} \sigma / \mathrm{d} \varepsilon$ and $\varepsilon_{\mathrm{f}}$ induced by the low dose of $0.043 \mathrm{MGy}$-HLEBI are explained by compressive force and stress relaxation induced by the coulomb repulsive force near dangling bonds for silica glass with interatomic distance and coordination number estimated by radial distribution function. ${ }^{27)}$ For UHMWPE (present work), the situation is different because the amorphous phase is above its glass transition temperature is in the rubbery state. Thus, there is no chance that HLEBI may change the density of this phase as any internal stresses would relax in very short times. However, the crystalline phase may be damaged by electron irradiation, which could change its average density with possible slight variations on 
mechanical behavior. Further studies would be necessary to check such hypothesis.

One can observe that the low dose of 0.043 MGy-HLEBI at room temperature remarkably increases the elastic properties (yield stress and Young modulus) and slightly increases the fracture strain and tensile strength, as shown in Fig. 1(a). 0.043 MGy-HLEBI decreases the degree and increases width of $\mathrm{X}$ ray peaks, as shown in Fig. 3 .

Additional dose from 0.043 to 0.43 MGy does not change the Young modulus, which is again consistent with the crystallinity ratio decrease from 46 to $43 \%$. The same trend can be seen for the yield stress. On the other hand, the strain at break is essentially decreasing with HLEBI dose likely because of the average chains length decrease. The melting temperature is observed to decrease when the HLEBI dose from 0.22 to $0.43 \mathrm{MGy}$ increases. ${ }^{19)}$ Additional dose of 0.43 MGy-HLEBI decreases the melting temperature, by 4 degrees. The melting temperature decrease is related to the decreasing of crystal periodicity, and is consistent with the polymer degradation as discussed above.

Compared with untreated sample, the additional high dose of $0.43 \mathrm{MGy}-\mathrm{HLEBI}$, which induces the radiation damages, not only decreases the melting point in Fig. 4 and Table 1, but also decreases and increases the degree and width of $\mathrm{X}$ ray peaks in Fig. 3, respectively. Consequently, the additional dose enlarges the mean atomic distance and decreases the periodicity, which is lower than for the untreated samples. The radiation damages are generated by additional density of active terminated atoms with dangling bonds, when HLEBI cuts polymers.

In addition, when increasing again the HLEBI dose the crystallinity cannot increase anymore, probably because of the presence of new crosslinking nodes induced by irradiation, though the damage due to chain scission still increase. As a consequence the irradiated zones become very brittle and cannot follow the deformation of the safe zone, leading to a very weak mechanical behavior. The tensile strength and fracture strain of 0.22 MGy-HLEBI are in data obtained for low and high doses of HLEBI.

Above the melting point the irradiation effect is strongly negative on the mechanical behavior, as shown in Fig. 1(b). Since the hot-press temperature of $423 \mathrm{~K}$ is higher than melting point, strengthening by homogeneous distribution of atomic scale expansion sites is not effective, resulting in reducing ductility. This can be also explained because the HLEBI has led to a decrease of chain length inducing a strong creep during the tensile test. These observations strongly suggest that the crystallinity ratio is the key parameter, which is able to explain the improvement of properties due to HLEBI.

\section{Conclusion}

Effects of low voltage electron beam irradiation (HLEBI) from 0.043 to $0.43 \mathrm{MGy}$, performed prior to sintering, on the tensile strength $\left(\sigma_{\mathrm{b}}\right)$, fracture strain $\left(\varepsilon_{\mathrm{f}}\right)$, yield strength $\left(\sigma_{\mathrm{y}}\right)$ and its Young modulus $(\mathrm{d} \sigma / \mathrm{d} \varepsilon)$ were investigated for UHWMPE sintered with crystallinity ration of $37 \%$.

(1) Experimental results showed the low dose of 0.043 MGy enhanced the crystallinity. Additional dose up to 0.43 MGy slightly decreases the crystallinity and crystal size probably because of the increase of chain defects density, like crosslinking nodes.

(2) For low doses, the HLEBI doses enhanced the Young modulus $(\mathrm{d} \sigma / \mathrm{d} \varepsilon)$ and yield stress, which is consistent with the increase in the crystallinity ratio from 37 to $46 \%$. The strain at break strongly decreases with HLEBI dose due to the average decrease of macromolecules length. Moreover the mechanical behavior above the melting point shows that HLEBI has led to a decrease of the chain length leading to a drastic creep and/or early fracture during tensile tests.

\section{Acknowledgements}

This work was partly supported by the JSPS Core-to-Core Program, A. Advanced Research Networks, "International research core on smart layered materials and structures for energy saving".

\section{REFERENCES}

1) P. J. Flory and T. G. Fox: J. Am. Chem. Soc. 73 (1951) 1904-1908.

2) P. G. de Gennes: J. Chem. Phys. 55 (1971) 572-579.

3) D. Jauffrès, O. Lame, G. Vigier and F. Doré: Macromolecules 41 (2008) 9793-9801.

4) D. Jauffrès, O. Lame, G. Vigier and F. Doré: Polymer 48 (2007) 63746383.

5) T. Deplancke, O. Lame, F. Rousset, I. Aguili, R. Seguela and G. Vigier: Macromolecules 47 (2014) 197-207.

6) Y. Nishi, H. Izumi, J. Kawano, K. Oguri, Y. Kawaguchi, M. Ogata, A. Tonegawa, K. Takayama, T. Kawai and M. Ochi: J. Mater. Sci. 32 (1997) 3637-3639.

7) A. Kasashima, N. Iwataka, J. Kawano, N. Honda and Y. Nishi: J. Adv. Sci. 9 (1997) 70-71.

8) K. Oguri, N. Iwataka, A. Tonegawa, Y. Hirose, K. Takayama and Y. Nishi: J. Mater. Res. 16 (2001) 553-557.

9) K. Oguri, K. Fujita, M. Takahashi, Y. Omori, A. Tonegawa, N. Honda, M. Ochi, K. Takayama and Y. Nishi: J. Mater. Res. 13 (1998) 3368-3371.

10) K. Oguri, N. Iwatani, H. Izumi, A. Tonegawa, K. Takayama and Y. Nishi: Proc. of 2nd JAPAN-FRANCE SEMINAR and Intelligent Materials and Structures, (University of Louis Pasteur Strasbourg, France, 1998) pp. 142-144.

11) A. Mizutani and Y. Nishi: Mater. Trans. 44 (2003) 1857-1860.

12) T. Takahashi, T. Morishita and Y. Nishi: J. Japan Inst. Metals 69 (2005) 759-762.

13) R. Christenhusz and L. Reimer: Z. Angew. Phys. 23 (1967) 396-404.

14) W. F. Libby: Anal. Chem. 19 (1947) 2-6.

15) M. Kanda, K. Yuse, B. Guiffard, L. Lebrun, Y. Nishi and D. Guyomar: Mater. Trans. 53 (2012) 1806-1809.

16) C. W. Bunn: Trans. Faraday Soc. 35 (1939) 482-491.

17) E. Epacher, J. Tolveth, C. Krohnke and B. Pukanszky: Polymer 41 (2000) 8401-8408.

18) D. J. Dijkstra and A. J. Pennings: Polymer Bull. 17 (1987) 507-513.

19) J. D. Hoffmann, G. T. Davis and J. I. Lauritzen, The Rate of Crystallization of Linear Polymers with Chain Folding, Vol. 3 (Plenum Press, 1976) pp. 497-614.

20) A. Mizutani and Y. Nishi: Mater. Trans. 44 (2003) 1857-1860.

21) T. Takahashi, T. Morishita and Y. Nishi: J. Japan Inst. Metals 69 (2005) 759-762.

22) H. Sato, Y. Inoue, K. Iwata, A. Tonegawa and Y. Nishi: J. Japan Inst. Metals 72 (2008) 520-525

23) Y. Nishi and K. Iwata: Mater. Trans. 46 (2005) 2241-2245.

24) Y. Nishi and K. Iwata: Mater. Trans. 47 (2006) 1810-1814.

25) K. Iwata and Y. Nishi: Mater. Trans. 49 (2008) 2058-2062.

26) K. Iwata and Y. Nishi: Mater. Trans. 51 (2010) 121-127.

27) N. Yamaguchi, K. Oguri, A. Tonegawa and Y. Nishi: J. Japan Inst. Metals 68 (2004) 198-201. 